\title{
PLANTAS CONSIDERADAS DANINHAS PARA CULTURAS \\ COMO FONTES DE NÉCTAR E PÓLEN
}

\author{
MITZI BRANDÃO 1/ \\ MANUEL LOSADA GAVILANES-2/ \\ LÚCIA HELENA DE SOUZA CUNHA - $3 /$ \\ JÚLIO PEDRO LACA- 4/ \\ CYNTHIA CARDOSO- 5/

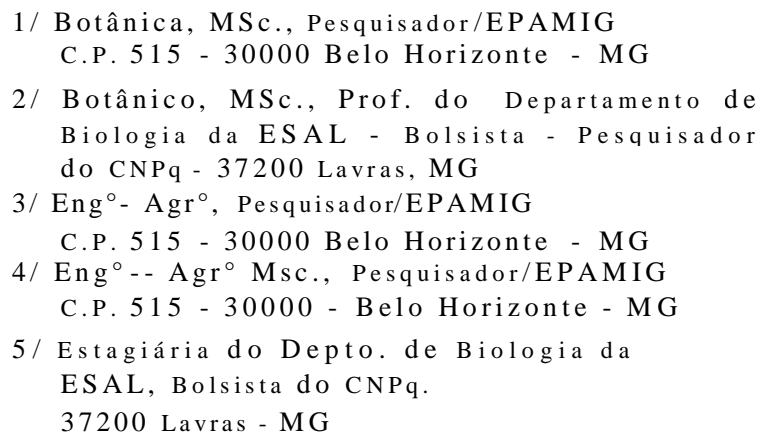

\section{RESUMO}

São relacionadas 164 espécies de plantas consideradas daninhas às culturas, no Estado de Minas Gerais, e que são produtoras de néctar e pólen.

Essas plantas poderiam ser exploradas economicamente, visan do o fornecime nto de matéria prima a apicultura, e como fonte de alimento para os inse tos polinizad ores.

PALAVRAS-CHAVE: Plantas Danin has, Polinização, Néctar, Pólen .

\section{SUMMARY}

PLANT SPECIES CONSIDERED WEEDS AS SOURCE OF NECTAR AND POLLEN

There are related 164 species of weed plants of cultures in the state of Min as Gerais as source of nectar and nectar and pollen.

These plants could be used economically for the purpose of supply of raw material to the apiculture and as source of food for the pollination ins ects.

KEY -WOR DS: Weeds, Pollination, Nectar.

\section{INTRODUÇÃO}

Des de 1972, den tro do projeto lig ado à área de plantas daninhas, tem sido intensivas as coletas no Estado de Minas Gerais. Inicialmente, esse levantamento foi realizado por cultura, nas áreas tradicionalmente ligadas aos produtos 
de interesse. Foram visitadas nessa ocasião as fazendas mais representativas em cada cultura, sendo os resultados obtidos já relatados em Ferreira \& Laca Buendia (4). Dando cobertura a projetos ligados a outras linhas de pesquisas, pontos mais distantes do Estado, foram visitados, em pequenas e medias propriedades (1), assim como aquelas inclusas nas formações naturais do Estado (5). As obse rvações a nível de campo, daquelas plantas daninhas produtoras de nectar e pólen, foram pois acumulando-se com o decorrerdos anos.

Algumas plantas daninhas estuda das no presente trabalho, foram mencionadas por outros autores(1,2, 3, $4,6,7,8,9,10)$.

O presente trabalho cataloga as plantas daninhas que fornecem nectar e/ou pólen, assim como a época da floração e ilustração de algumas estruturas que forn ecem nectar ou pólen.

\section{MATERIAIS E MÉTODOS}

A listagem e as épocas de floração foram obtidas quando dos trabalhos anteriormente relacionados.

Posteriormente, foram coletadas flores daquelas espécies comumente encontradas, objetivando-se a confecção de figuras ilustrativas, apresentadas em anexo.

Os órgãos florais de plantas poliníferas, nectaríferas e pólen-nectaríferas foram desenhados (figuras 1 a 9).
O material coletado acha-se incluído no PAMG e na ESAL, herbários da Empresa Agropecuária de Minas Gerais e da Escola Superior de Agricultura de Lavras, respectiva mente.

\section{RESULTADOS E DISCUSÃO}

$\mathrm{Na}$ Tabela 1, encontra-se a listagem de espécies de interesse como fontes de nectar e/au pólen, onde pode-se observar que foram levantadas 164 espécies, das quais, 29 flore cem todo o ano, sendo que 9 espécies fornecem pólem: Althernanthera brasiliana (L.) Kuntze.; Commelina erecta L., Tradescantia elongata Meyer, Bidens pilosa L., Momordica charantia L., Ricinus communis L., Cassia alata L., Mimosa pudica L., e Hedychium coronarium Koening., 12 espé cies fornecem nectar: Cordia verbe nacea D.C., Euphorbia brasi liens is Lam. Euphorbia heterophyll a L., Euphorbia pilulifera L., Euphorbia prostata Ait., Stylosanthes guyanen sis (Aubl.) Sw., Stylosanthes scabra Vog., Portulaca oleracea L. Bor reira verticillata (L.) G.F. Meyer, Starchytarphetta cayennensis (L. C. Rich) Vahl., e Starchytarphetta polyura Schaner, e 8 espécies que for necem nectar e pólen: Ascle pia curassavica L., Mikania cordifolia Willd., Veronia remotiflora Rich., Vernonia scorpioides (lam.) Pers., Wedelia paludosa D.C.Leono $^{\text {tis }}$ nepetaefolia (L.) R.Br., Leonu rus sibiricus L., e Mars ypianthes chamaedrys (Vahl.) O. Kuntze. Estas espé cies pertecem a 92 gêneros engloba- 
TABELA 1 - Relação da famílias, espécies e respectívos nomes populares das plantas daninhas, ocorrentes en Minas Gerais, com pøssibilidade de serem utilizadas como fonte de néctar e/ ou pólen

\begin{tabular}{|c|c|c|c|c|c|}
\hline FAMÍLIA & NOME CIENTÍFICO & NOME (S) POPULAR (RES) & $\begin{array}{l}\text { EPOCAS DE } \\
\text { FLORAÇAO }\end{array}$ & NECTAR & POLEN \\
\hline Acanthaceae & Thumbergia alata Bojer. & Amarelinha, maria-sen-vergorha & set/maio & & $\mathrm{x}$ \\
\hline Amarenthaceee & Alternanthera brasiliana (L.) Kuntze & Cabeça-branca & todb ano & & $\mathrm{x}$ \\
\hline Anecantiaceae & Schinus terebentifolius Raddi. & srceirirha & set/maio & $\mathrm{x}$ & \\
\hline Apocynaceae & Peschiera fuchsiaefolia Miers. & Leiteiro & ago/dez & $\mathrm{x}$ & \\
\hline Asclepiadacear & Asclepia curassavica L. & Oficial-de-sala & todb an & $\mathrm{x}$ & $\mathrm{x}$ \\
\hline Bignoniaceee & Pyrostegia venusta Miers. & Cipö-de-São Jä̃o & maio/ago & $\mathrm{x}$ & $x$ \\
\hline 3orraginaceae & Cordia curassavica Roem. et Schult. & Funo-bravo & set/maio & $\mathrm{x}$ & \\
\hline · & Cordia vertenacea Dc. & Funo-bravo, casaudinho & rodo ano & $\mathrm{x}$ & \\
\hline \multirow[t]{2}{*}{ Japparideceee } & Cleame affinis Dc. & Mussarbé & aut/dez & & $\mathrm{x}$ \\
\hline & Clecone spinosa L. & Mussembezinho, unbuzinho & dez/ahril & & $\mathrm{x}$ \\
\hline \multirow[t]{3}{*}{ Vommelineceae } & Commelina diffusa Bur. & Trapoeraba, trapoeiraba & raio/jultho & & $\mathrm{x}$ \\
\hline & Cormelina erecta $L$. & Trapoeraba & todb ano & & $\mathrm{x}$ \\
\hline & Tradescantia elongata Meyer & Trapoeraba & todo ano & & $\mathrm{x}$ \\
\hline \multirow[t]{2}{*}{ Taryophyllaceee } & $\begin{array}{l}\text { Spergula arversis } \mathrm{L} . \\
\text { Stellaria media (L.) Cyrill. }\end{array}$ & $\begin{array}{l}\text { Pega-pinto, espérgula } \\
\text { Estérgula,morriäo-dos-pessari- }\end{array}$ & set/maio & & $\mathrm{x}$ \\
\hline & & mos & set/maio & & $\mathrm{X}$ \\
\hline \multirow[t]{6}{*}{ 'onpositae } & Acanthospermul australe (Loef.)O.Kuntze & $\begin{array}{l}\text { Carrapicho, carrapicho-de-carnei- } \\
\text { ro,carrepicho-manoto,maroto }\end{array}$ & março/maio & $\mathrm{x}$ & $x$ \\
\hline & Actyrocline satureioides & Macela & maio/agosto & & $\mathrm{x}$ \\
\hline & Ageratam coryzondes $\mathrm{L}$. & Erva-Säo Jaẽo, mentrasto & maio/egosto & & $\mathrm{X}$ \\
\hline & Antrosia polystachia DC. & $\begin{array}{l}\text { Artendsia-brava, cravorana, losna- } \\
\text { do-campo, losna-brava }\end{array}$ & jan/margo & & $x$ \\
\hline & Beccharis dracunaulifolia DC. & Vassourirha, veseoura, alecrin & abril/agosto & $\mathbf{X}$ & $x$ \\
\hline & Beccharis genistelioides $D$. & Carqueja & abri/agosto & $\mathbf{x}$ & $\mathrm{x}$ \\
\hline
\end{tabular}


TABEIA 1 - Cont...

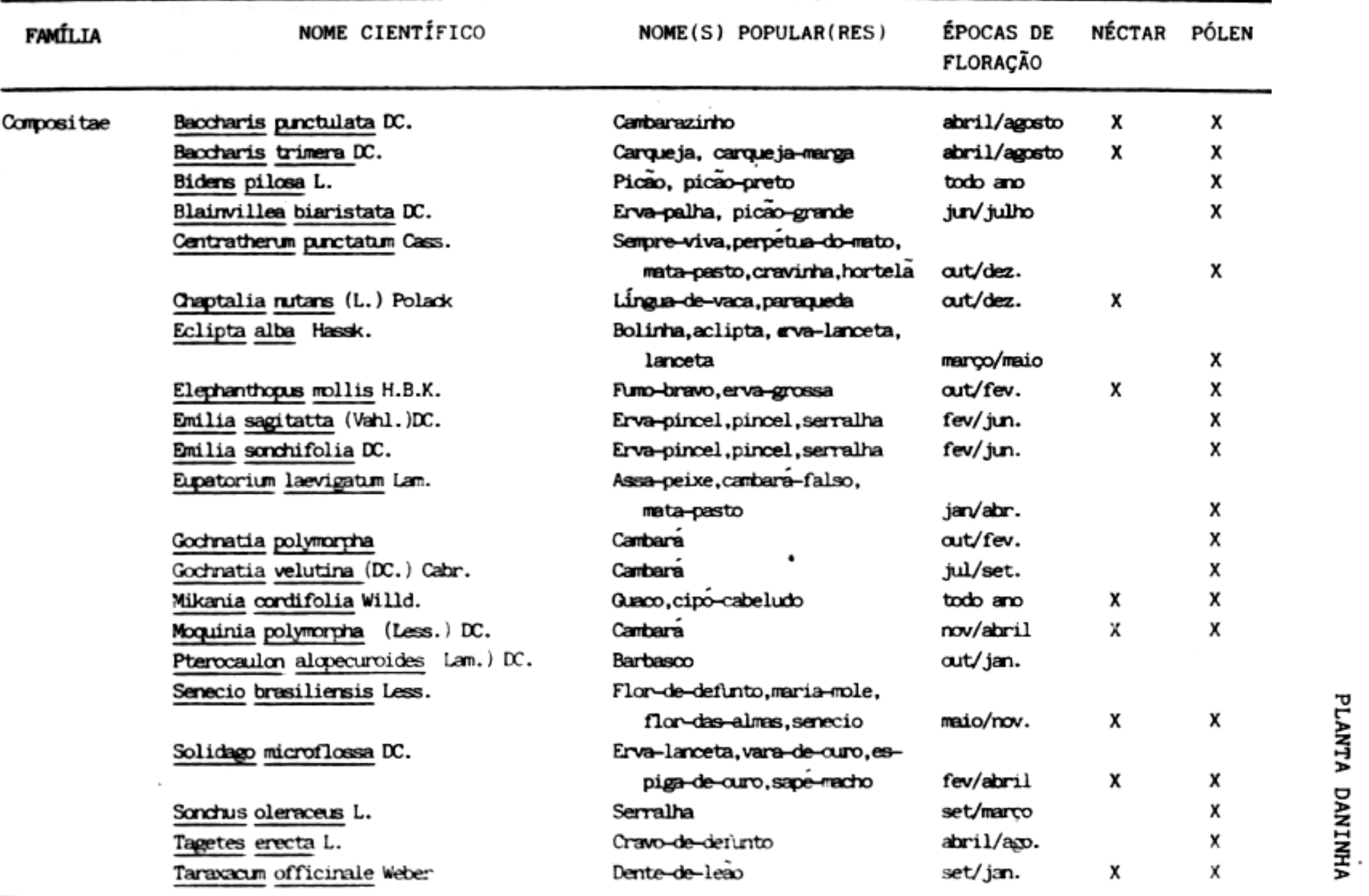


TABBIA 1 - Cont...

\begin{tabular}{|c|c|c|c|c|c|}
\hline FAMÍLIA & NOME CIENTÍFICO & NOME (S) POPULAR(RES) & $\begin{array}{l}\text { ÉPOCAS DE } \\
\text { FLORAÇÃOO }\end{array}$ & NÉCTAR & PÓLEN \\
\hline \multirow[t]{11}{*}{ Compositae } & Trixis antimenorthea (Sctrank.) Mart. & Solidania & Jul/set. & $x$ & $\mathbf{x}$ \\
\hline & Vernania aurea Mart. & Assa-peixe & set/dez. & $\mathrm{x}$ & $\mathbf{x}$ \\
\hline & Verrania difusa Less. & Assa-peixe & set/dez. & $\mathrm{x}$ & $\mathbf{x}$ \\
\hline & Vernania ferruginea Less. & Assa-peixe & ago/nov. & $x$ & $x$ \\
\hline & Vernonia grandiflora Less. & Assa-peixe,mata-pasto & ago/nov. & $x$ & $x$ \\
\hline & Vernania nudiflora Less. & Alecrim & ago/nov. & $x$ & $x$ \\
\hline & Vernania polyanthes Less. & $\begin{array}{l}\text { Assa-peixe, assa-peixe-branco, } \\
\text { cenbará-branco }\end{array}$ & maio/ago. & $x$ & $\mathrm{x}$ \\
\hline & Vernonia remotiflara Rich & Roxima & todo ano & $x$ & $x$ \\
\hline & Vernonia scorpioides (Lan.) Pers. & Erva-Säo-Simëo, raxima & todo ano & $x$ & $x$ \\
\hline & Vernonia westiniana Less. & Assa-peixe & set/dez. & $x$ & $\mathrm{x}$ \\
\hline & Wedelia paludosa DC. & Margaridinha & todo ano & $x$ & $\mathrm{X}$ \\
\hline \multirow[t]{3}{*}{ Convolvulaceae } & Ipornoea cairica (L.) Sweer. & Corda-de-viola, getirana & set/julho & & $x$ \\
\hline & Ipomoea purpurea Lam. & $\begin{array}{l}\text { Companhia, conda-de-viola, } \\
\text { bon-dia }\end{array}$ & maio/agosto & $\mathrm{x}$ & \\
\hline & Merremia macrocalyx (Ruiz et Pav. ID'Do, & $\bullet$ & fev/jurino & & $x$ \\
\hline \multirow[t]{3}{*}{ Cruciferae } & Brassica campestris L. & Mostarda-brava, mostanda-crespa & maio/ago. & $\mathrm{x}$ & $x$ \\
\hline & Raphanus raphanistrum L. & $\begin{array}{l}\text { Nabo, nabiça, nabo-bravo, rabene- } \\
\text { te-selvagem }\end{array}$ & jul/ago. & $\mathrm{x}$ & $\mathbf{X}$ \\
\hline & Sinapsis arvensis $\mathrm{L}$. & Mostarda, mostanda-silvestre & maio/julho & $\mathrm{x}$ & \\
\hline \multirow[t]{2}{*}{ Qucurbi taceae } & Luffa cylindrica & Bucha, bucha-vegetal & set/maio & & $x$ \\
\hline & Momordica charantia L. & Melão-de-São Caetano & tado ano & & $\mathrm{x}$ \\
\hline \multirow[t]{3}{*}{ Euphorbiaceae } & Crotan campestris St.Hil. & Velame & abril/jurho & $\mathrm{x}$ & \\
\hline & Euphortia brasiliensis Lar. & Erva-andorinha, leiteira & todb ano & $\mathrm{x}$ & \\
\hline & Euphorbia heteroodylla L. & Leiteira & todo ano & $\mathrm{x}$ & \\
\hline
\end{tabular}


TABEIA 1 - Cont.

\begin{tabular}{|c|c|c|c|c|c|}
\hline FAḾ́LIA & NOME CIENTIFICO & NOME (S) POPULAR (RES) & $\begin{array}{l}\text { ÉPOCAS DE } \\
\text { FLORAÇÃO }\end{array}$ & NÉCTAR & PÓLEN \\
\hline \multirow[t]{3}{*}{ Ephorbiaceae } & Quphorbia pilulifera L. & Erva-de-Santa Luzia, leiteira & todb ano & $\mathrm{x}$ & \\
\hline & Eyphorbia prostrata Ait. & Quebra-pedra-rasteino & todb ano & $\mathrm{x}$ & \\
\hline & Ricins comminis L. & Mamona, carrapateira & todb ano & & $\mathrm{x}$ \\
\hline \multirow[t]{3}{*}{ Gramineae } & Cymodan dactylan (L.) Pers. & Grama-seda,capim-de-burro & maio/jurho & & $\mathrm{x}$ \\
\hline & Paspalum notatum Flitgge & Batatais, grame-batatais & dez/jan. & & $x$ \\
\hline & Melinis minutiflara Bearv. & Meldio, capim-meloso,gondura & abril/julho & & $x$ \\
\hline \multirow[t]{12}{*}{ Labiatae } & Hyptis brevipes Poit. & Malva-de-cheiro,hortelä-do-campo & abril/julho & $\mathrm{x}$ & \\
\hline & Hyptis Iophanta Mart. ex. Benth. & Cidreira,hortelä,hortelä-do-canpo & o abril/julho & $\mathrm{x}$ & \\
\hline & Hyptis nudicaulis Benth. & Cidreira, hortelä, vareta & abrill/julho & $x$ & \\
\hline & Hyptis pectinata Poit. & Cidreira, hortelä, vareta & março/julho & $x$ & \\
\hline & $\begin{array}{l}\text { Hyptis suaveolens Poit. } \\
\text { Leanotis nepetaefolia (L.)R.Br. }\end{array}$ & $\begin{array}{l}\text { Cheirosa,menta,hortelä-da-campo } \\
\text { Cordäa-de-S.Francisco, cordäo-de- }\end{array}$ & marco/julho & $x$ & \\
\hline & & frade & todo ano & $x$ & $\mathrm{x}$ \\
\hline & Leanurus sibirias $\mathrm{L}$. & Erva-macaẽ, macaé, rubim, rabo-de- & & & \\
\hline & & gato,pasto-de-abelha, rubim & todb ano & $\mathrm{x}$ & $x$ \\
\hline & Leucas martinicersis R.Br. & Cordäo-de-frada & set/dez. & $\mathrm{X}$ & $\mathrm{x}$ \\
\hline & Marsypianthes chamaedrys (Vahl.) O.Kuntze & Menta, vassaura, hartelã & todo ano & $x$ & $\mathrm{x}$ \\
\hline & Stachys arversis $L$ & Hortelã-das-roças, orelha-de-urso, & & & \\
\hline & & urtiga-mensa & set/dez. & & $\mathrm{x}$ \\
\hline \multirow[t]{4}{*}{ Leguminosae } & Acacia banariensis Gil. & Unha-de-gato & set/fev. & $x$ & $\mathrm{x}$ \\
\hline & Acacia peniculta willd. & Unha-de-gato & dez/fev. & $\mathrm{x}$ & $x$ \\
\hline & Acacia plumosa Lave & Arranha-gato & set/fev. & $\mathrm{x}$ & $\mathrm{x}$ \\
\hline & Cassia alata L. & Fedegoso-grande & todo ano & & $x$ \\
\hline
\end{tabular}


TABEIA 1 - Cont...

\begin{tabular}{|c|c|c|c|c|c|}
\hline \multirow[t]{4}{*}{ Leguminosae } & Cassia bahinaefolia Kunt. & Coraçäo, erva-coraçäo & set/maio & & $\mathrm{x}$ \\
\hline & Cassia flexussa $L$ & $\begin{array}{l}\text { Fedegoso-folha-miuda, mata-pesto, } \\
\text { pena-de-agalinha }\end{array}$ & fev/março & & $\mathrm{x}$ \\
\hline & Cassia ocidentalis $\mathrm{L}$. & Fedegoso, mata-pasto, tararuau & set/nov & & $\mathrm{x}$ \\
\hline & Cassia patellaria $\mathrm{DC}$. & Falsa-dormideira, pena-de-galinha & set/dez. & & $\mathrm{x}$ \\
\hline \multirow{2}{*}{$\stackrel{m}{g}$} & Cassia pubescens Jacq. & Fedegoso-do-mato & set/dez. & & $\mathrm{x}$ \\
\hline & Cassia rotandifolla Pers. & Fedegoso, erva-coraçäo & set/dez. & & $\mathrm{x}$ \\
\hline \multirow{4}{*}{ 刘 } & Cassia tora L. & Fedegoso,mata-pasto & jan/margo & & $x$ \\
\hline & Crotalaria anagyroides H.B.K. & Cortaläria, chocalho,guizo-de- & & & \\
\hline & & cascavél & aut/fev. & & $\mathrm{x}$ \\
\hline & Crotalaria incana L. & Crotalaria, chocalho, guizo-de- & art/fer. & & $\mathrm{x}$ \\
\hline \multirow{14}{*}{$\begin{array}{l}0 \\
0 \\
0 \\
0\end{array}$} & Crotolária lanceolata E.Mey & Crotalária, chocaltho & set/dez. & & $\hat{x}$ \\
\hline & Crotalária mucronata Desv. & Crotalária,chocalho,guiseiro & março/julho & $x$ & $\mathrm{x}$ \\
\hline & Crotalaria spectabilis Roth. & Chocalho-de-cascavél & set/jurino & $\mathrm{x}$ & $\mathrm{x}$ \\
\hline & Desmodium adscendens (Sw.) DC. & \multicolumn{3}{|c|}{ Carrapicho, cairapicho-beiço-de-boi set/jun. } & $\mathrm{x}$ \\
\hline & Desmodium barbatum (L.) Benth. & Barbedinho, carrapicho & set/dez. & & $\mathrm{x}$ \\
\hline & Desmodium discolor & Marmelada-de-cavalo & set/dez. & & $\mathrm{x}$ \\
\hline & Desmodium incanum $\mathrm{DC}$ & \multicolumn{3}{|c|}{ Carrapicho-beigo-de-boi, pega-pega set/dez. } & $\mathrm{x}$ \\
\hline & Desnocium purpureum(Mill.)Fawx.et Rend. & Pega-pega & $\mathrm{dez} / \mathrm{maio}$ & & $\mathrm{x}$ \\
\hline & Indigofera hirsuta $\mathrm{L}$ & Anil, anileira & jan/março & & $\mathrm{x}$ \\
\hline & Melilotus alba Lam. & Trevo-branco & set/maio & $\mathrm{x}$ & $\mathrm{x}$ \\
\hline & Melilotus indias (L.)Ait & Trevo-amarelo & set/maio & $\mathrm{x}$ & $\mathrm{x}$ \\
\hline & Mimosa invisa Mart. & Malicia,malicia-de-mulher & $\mathrm{fev} / \operatorname{mar} \varphi{ }^{\prime}$ & & $\mathrm{x}$ \\
\hline & Mimosa bimucronata DC. & Maricá & & & $x$ \\
\hline & Mimosa pudica L. & Dormideira,ralícia,sensitiva & todo ano & & $x$ \\
\hline
\end{tabular}


TABEIA 1 - Cont...

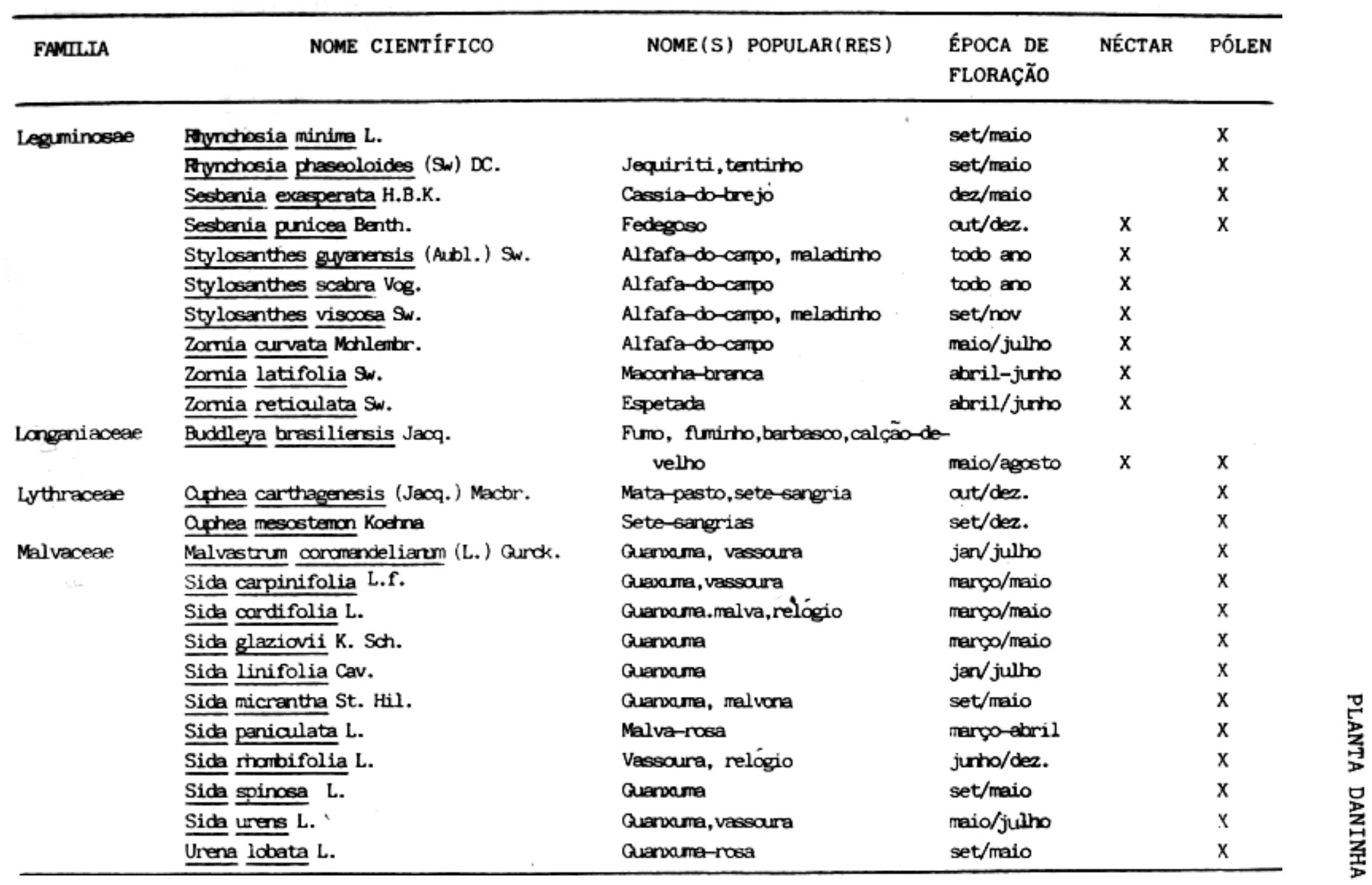


TABEIA 1 - Cont...

\begin{tabular}{|c|c|c|c|c|c|}
\hline FAMÍLIA & NOME CIENTfFICO & NOME (S) POPULAR(RES) & $\begin{array}{l}\text { ÉPOCAS DE } \\
\text { FLORAÇÃO }\end{array}$ & NÉCTAR & PÔLEN \\
\hline \multirow[t]{8}{*}{ Oxalidaceae } & Oxal is bipartita St. Hil. & Azedinha, trevo & set/dez. & & $\mathrm{x}$ \\
\hline & Oxalis comioulata L. & Azedima, trevo & set/dez. & & $x$ \\
\hline & Oxalis corymbosa DC. & Azedinha, trevo & set/dez. & & $\mathbf{x}$ \\
\hline & Oxalis hirsutissima Mart. et Zuoc. & Trevo-peludo & aut/dez. & & $\mathrm{x}$ \\
\hline & Oxalis martiniana $2 u c c$ & Azadinha, trevo & set/jan. & & $\mathrm{x}$ \\
\hline & Oxalis axyptera Prog. & Azedinha, trevo & set/jan. & & $\mathrm{x}$ \\
\hline & Oxalis refracta St. Hil & Azedinha, trevo & dez/maio & & $\mathrm{x}$ \\
\hline & Oxalis barretoi & & & & \\
\hline \multirow[t]{2}{*}{ Polygonaceae } & Fagopyrum esculentum Moench. & Trigo-sarraceno & dez/maio & & $\mathrm{x}$ \\
\hline & Polyganm acre H.B.K. & Erva-de-bicho & abril/jurho & $x$ & $\mathrm{x}$ \\
\hline \multirow[t]{2}{*}{ Pontederiaceae } & Eichhomia arurea Kunt. & Aguapé & set/marco & $x$ & $x$ \\
\hline & Eichiomia crassipes (Mart. ISolms & Aguapé & set/março & $\mathrm{x}$ & $x$ \\
\hline \multirow[t]{2}{*}{ Portulacaceae } & Portallaca grandiflara Hook. & Beldroega,beldroega-grande & set/marco & $\mathrm{x}$ & $\mathrm{x}$ \\
\hline & Portulaca olerace $\mathrm{L}$ & Beldroega, ora-pro-nobis & todo ano & $\mathrm{x}$ & \\
\hline Rubiaceae & Borreria verticillata (L.) G.F.Meyer & $\begin{array}{l}\text { Estrelinha, quebra-tijela, vassar } \\
\text { ra-branca . }\end{array}$ & todo ano & $x$ & \\
\hline \multirow[t]{2}{*}{ Sapindaceae } & Serjanea erecta & Cipö-timbó, timbó & set/maio & $\mathrm{x}$ & \\
\hline & Serjanea gracilis Radlk. & Cipó-timbó, timbó & set/maio & $\mathrm{x}$ & \\
\hline \multirow[t]{3}{*}{ Solanaceae } & Acristas arbarens $\mathrm{L}$. & Fruta-de-ponba, fruta-ie-galinha & set/nov & $\mathrm{x}$ & \\
\hline & Datura sueveolens willd. & Saia-branca, trmbeteira & set/maio & & $\mathrm{x}$ \\
\hline & Solanum peniculatum $\mathrm{L}$. & Junubebe & jan/abril & $\mathrm{x}$ & \\
\hline
\end{tabular}


TABEIA 1 - Cont...

\begin{tabular}{|c|c|c|c|c|c|}
\hline FAMÍLIA & NOME CIENTÍFICO & NOME (S) POPULAR (RES) & $\begin{array}{l}\text { ÉPOCAS DE } \\
\text { FLORAÇÃOO }\end{array}$ & NÉCTAR & PÓLEN \\
\hline \multirow[t]{2}{*}{ Sterauliaceae } & Melochia pyramidata & & nov/julho & $\mathbf{x}$ & $\mathrm{x}$ \\
\hline & Waltheria indica $\mathrm{L}$. & Malva-branca & abril/maio & $\mathbf{x}$ & $\mathrm{x}$ \\
\hline \multirow[t]{3}{*}{ Tiliaceae } & Carchorus hirtus L. & Vassaura & set/maio & $\mathbf{x}$ & $x$ \\
\hline & Triumfetta semitriloba Jaca. & $\begin{array}{l}\text { Carrepicho-de-calçach, malva } \\
\text { carrapicto }\end{array}$ & maio/set. & $\mathbf{x}$ & $\mathrm{x}$ \\
\hline & Triumfetta barthamia L. & Carrapichão, malva & maio/set. & $x$ & $\mathrm{x}$ \\
\hline Tumeraceae & Turnera ulmifolia L. & Xanana, chanana & set/maio & & $\mathrm{x}$ \\
\hline Typhaceae & Typha angustifolia $\mathrm{L}$. & Taboe & set/maio & & $x$ \\
\hline \multirow[t]{9}{*}{ Verbenaceae } & Lantana camara $\mathrm{L}$. & Canará, canbará, camara-de-espinho & & & \\
\hline & & milho-de-grilo & jan/jurho & $x$ & \\
\hline & Lantana lilacina Desf. & Canará-rosa & jan/jumo & $\mathrm{x}$ & \\
\hline & Lantana brasiliensis $\mathrm{L}$. & Camaré-branco & set/dez. & $\mathrm{x}$ & \\
\hline & $\begin{array}{l}\overline{\text { Lantana }} \text { nivee Vent. } \\
\text { Stactytarphetta cayernensis (L.C.Rich.) }\end{array}$ & Canará-branoo & set/dez. & $\mathrm{x}$ & \\
\hline & Vahl. & Gerväo-azul & todb ano & $\mathrm{x}$ & \\
\hline & Stachytarphetta polyura Schaner & Gervão & todb ano & $\mathrm{x}$ & \\
\hline & Verbena littaralis H.B.K. & Erva-de-S.Caetano & set/maio & $\mathrm{x}$ & \\
\hline & Verbera montevidensis Spr. & Amargosa & set/maio & $\mathrm{x}$ & \\
\hline Zingiberaceae & Hedychium coronarium Koening. & $\begin{array}{l}\text { Borboleta, lírio-do-brejo, bastäo- } \\
\text { de-Säo José }\end{array}$ & todb ano & & $x$ \\
\hline
\end{tabular}


TABELA 2 - Relação das Famílias enfocadas, números de gêneros e Espécies

\begin{tabular}{|c|c|c|}
\hline FAMILIA & GÊNERO & FSPÉCIES \\
\hline Acanthaceae & 1 & 1 \\
\hline Amaranthaceae & 1 & 1 \\
\hline Anacardiaceae & 1 & 1 \\
\hline Apocynaceae & 1 & 1 \\
\hline Asclepiadaceae & 1 & 1 \\
\hline Bignoniaceae & 1 & 1 \\
\hline Borraginaceae & 1 & 2 \\
\hline Capparidaceae & 1 & 2 \\
\hline Commelinaceae & 2 & 3 \\
\hline Caryophy 1 laceae & 2 & 2 \\
\hline Compositae & 25 & 30 \\
\hline Convolvulaceae & 2 & 5 \\
\hline Cruciferae & 3 & 3 \\
\hline Cucurbitaceae & 2 & 2 \\
\hline Euphorbiaceae & 3 & 7 \\
\hline Gramineae & 3 & 3 \\
\hline Labiatae & 6 & 10 \\
\hline Leguminosae & 11 & 37 \\
\hline Loganiaceae & 1 & 1 \\
\hline Ly thraceae & 1 & 2 \\
\hline Malvaceae & 3 & 11 \\
\hline Oxalidaceae & 1 & 8 \\
\hline Polygonaceae & 2 & 2 \\
\hline Pontederiaceae & 1 & 2 \\
\hline Portulacaceae & 1 & 2 \\
\hline Rubiaceae & 1 & 1 \\
\hline Sapindaceae & 1 & 2 \\
\hline Solanaceae & 3 & 4 \\
\hline Sterculiaceae & 2 & 2 \\
\hline Tiliaceae & 2 & 3 \\
\hline Turneraceae & 1 & 1 \\
\hline Typhaceae & 1 & 1 \\
\hline Verbenaceae & 3 & 8 \\
\hline Zingiberaceae & 1 & 1 \\
\hline
\end{tabular}



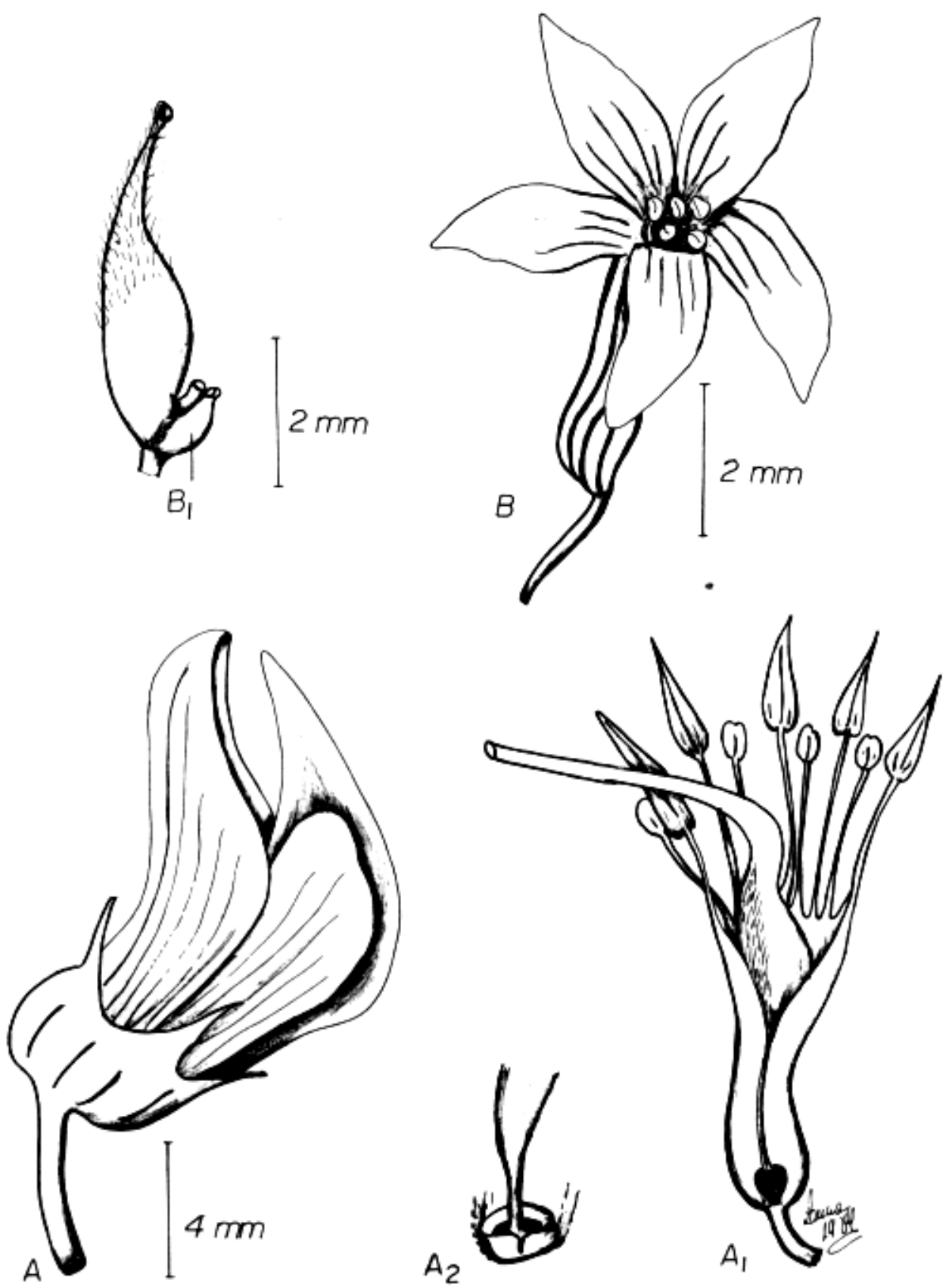

FIGURA I - Flores nectariferas - A - flor, $A_{1}$ - detalhe interno e $A_{2}$ base do ovário mostrando 0 nectário de Crotalaria mucronata, B - flor e $B_{1}$ nectários de Cuphea carthagenensis 


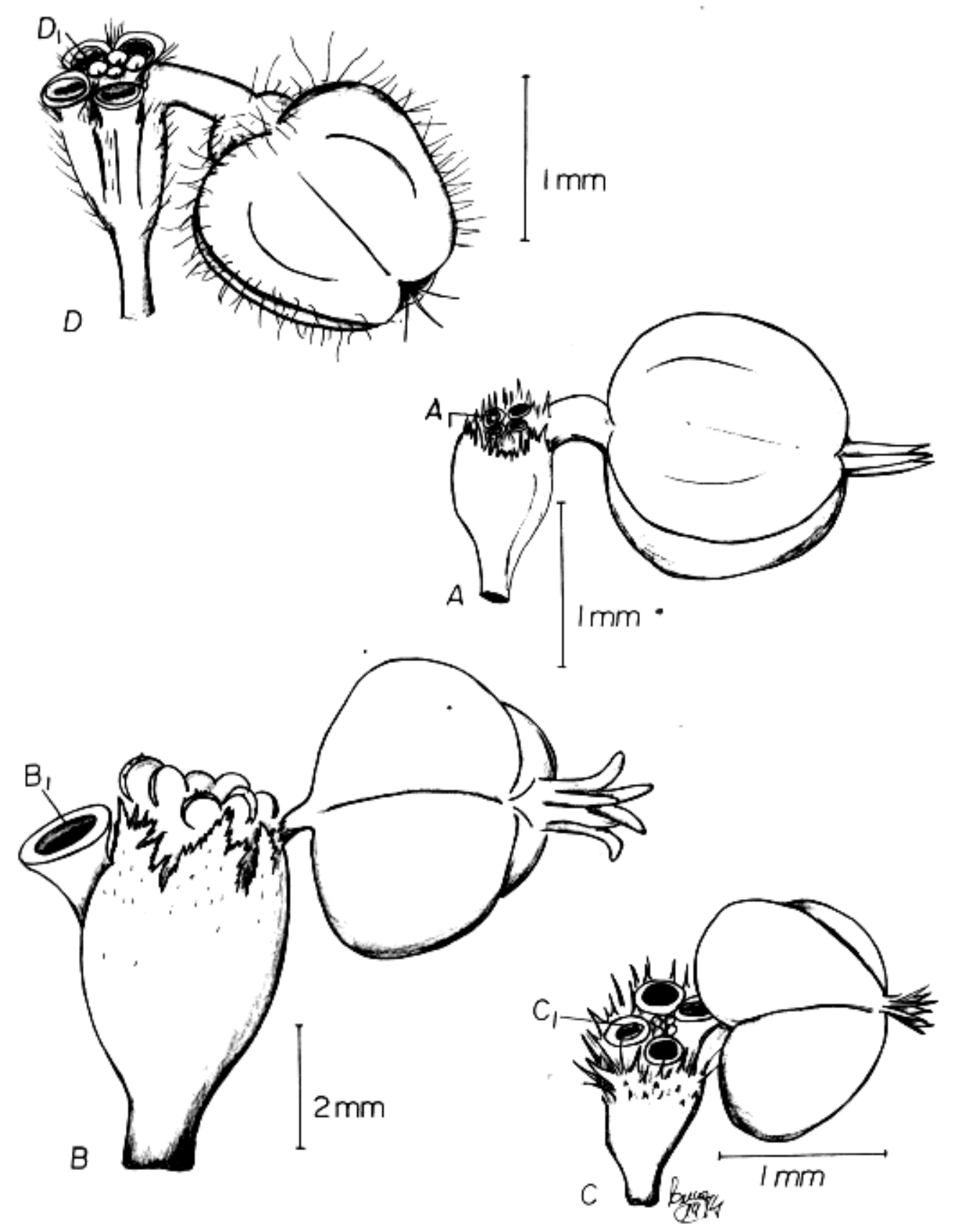

FIGURA 2 - Flores nectaríferas - A - inflorescência e $A_{1}$ - nectários de Euphorbia brasiliensis: $B$ - inflorescēncia e $B_{1}$ - nectário de Euphorbia heterophylla , C - inflorescência e $C_{1}$ - nectários de Euphorbia pilulifera D - inflorescência e $D_{1}$ - nectários de Euphorbia prostrata. 

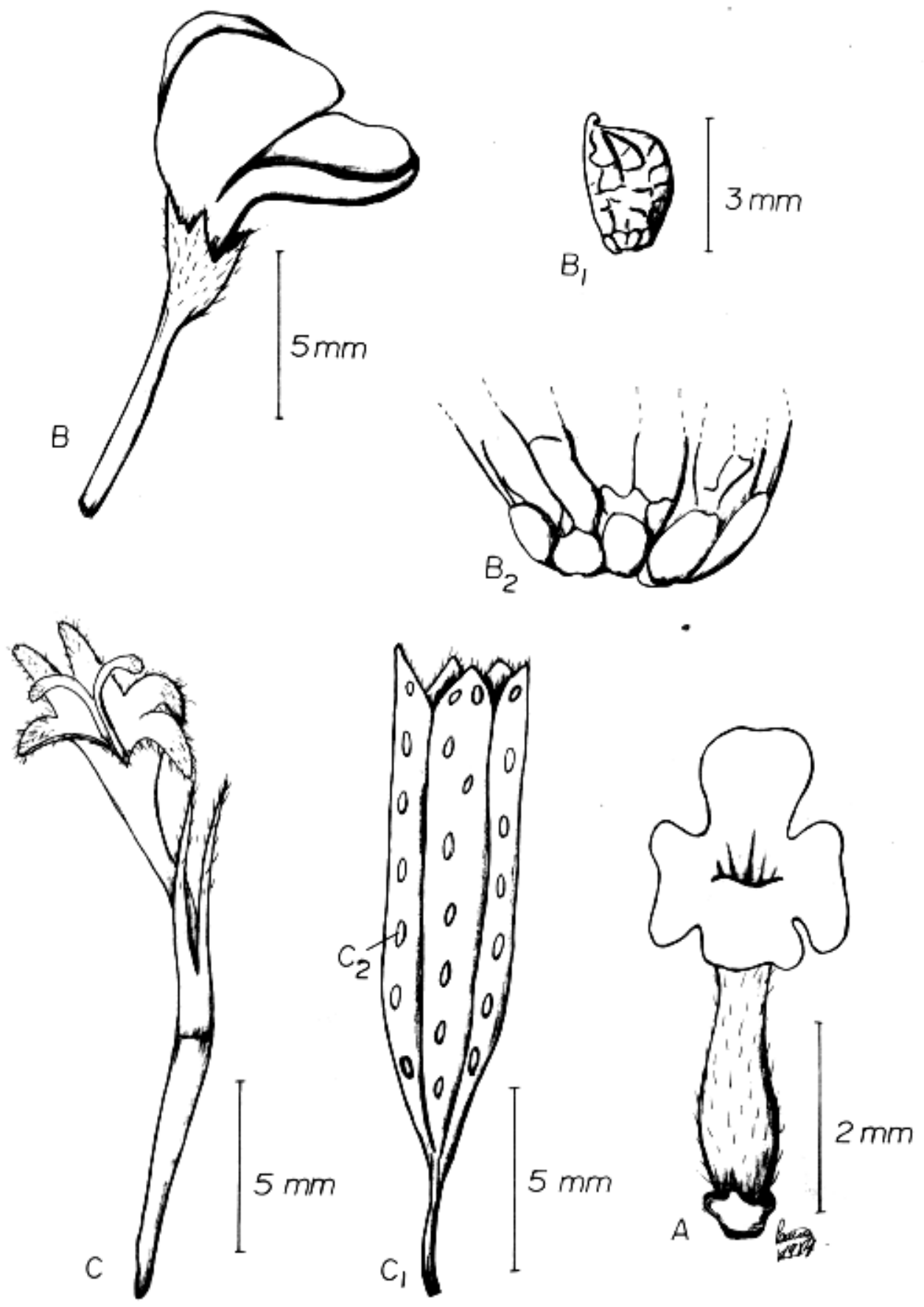
$\begin{aligned} \text { FIGURA } 3 \text { - Flores nectariferas - } A \text { - flor de } \frac{\text { Lantana }}{\text { brasiliensis, } B \text { - flor, } B_{1} \text { - semente }} & B_{2}-\end{aligned}$ detalhe dos nectários da semente de Stylosanthes guianensis, $\mathrm{C}$ - flor, $\mathrm{C}_{1}$ - bracteas e $\mathrm{C}_{2}$-nectários de Tagetes erecta. 


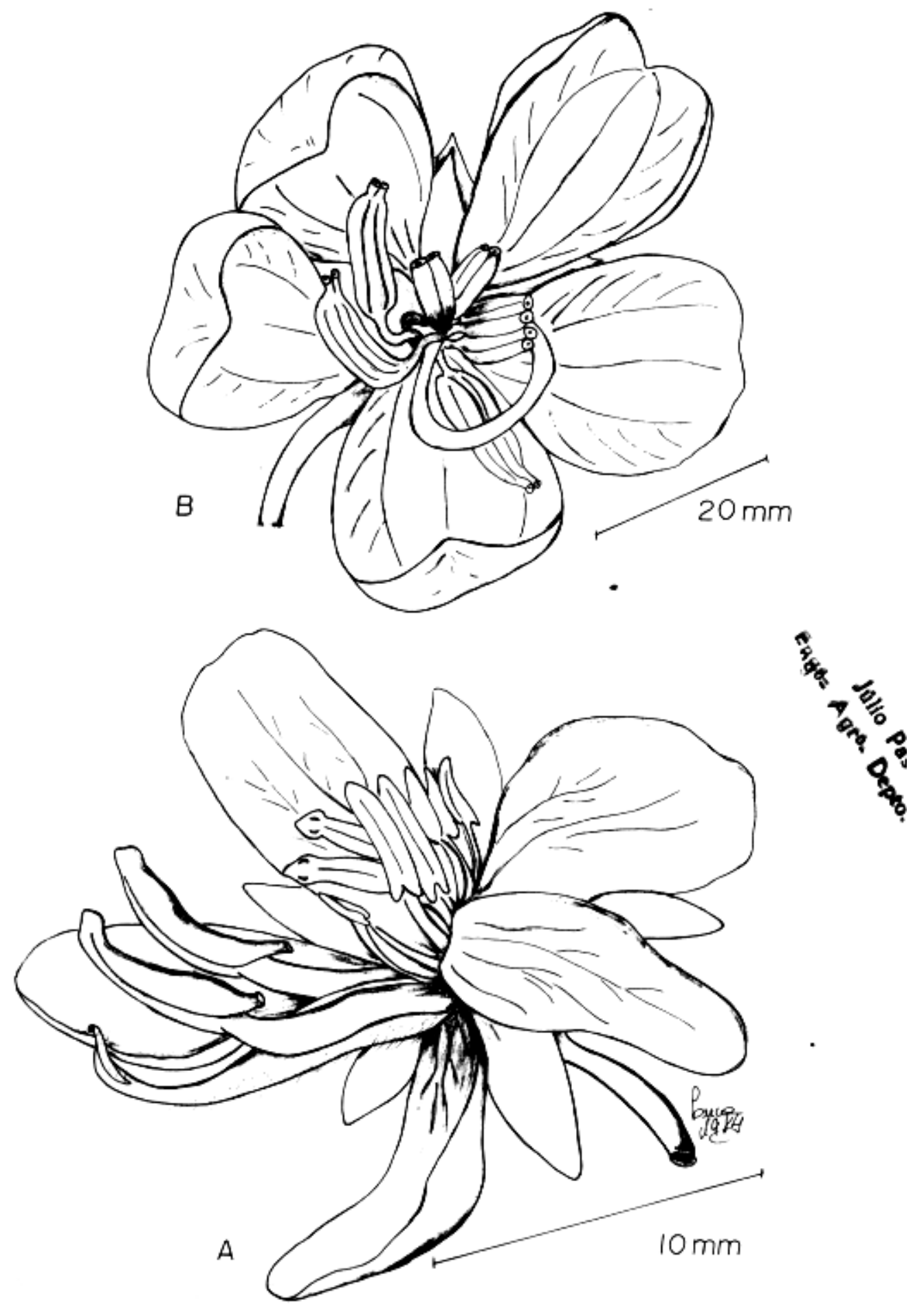

FIGURA 4 - Flores poliníferas - A - flor de Cassia occidentalis e B - flor de Cassia tora. 

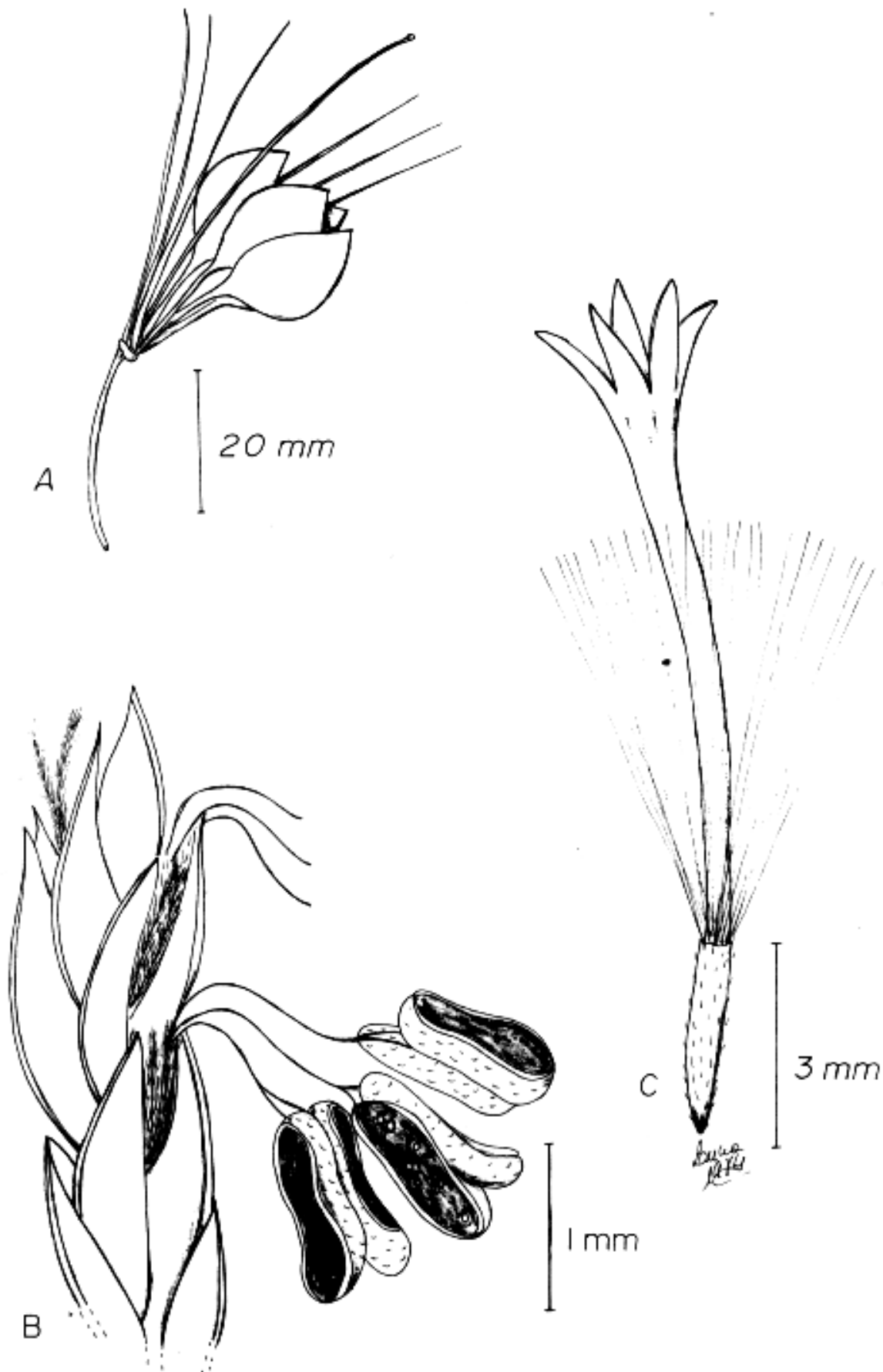

FIGURA 5 - Flores poliníferas - A - flor de Cleome spinosa ; B - detalhe da inflorescência de Cynodon dactylon e C - flor de Emilia sonchifolia. 


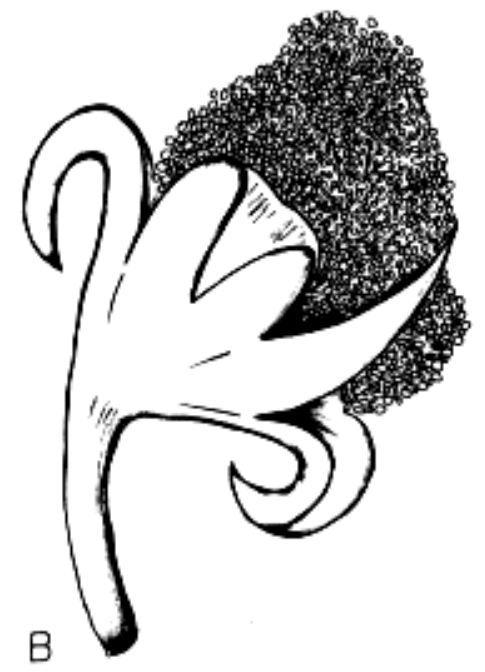

$4 \mathrm{~mm}$
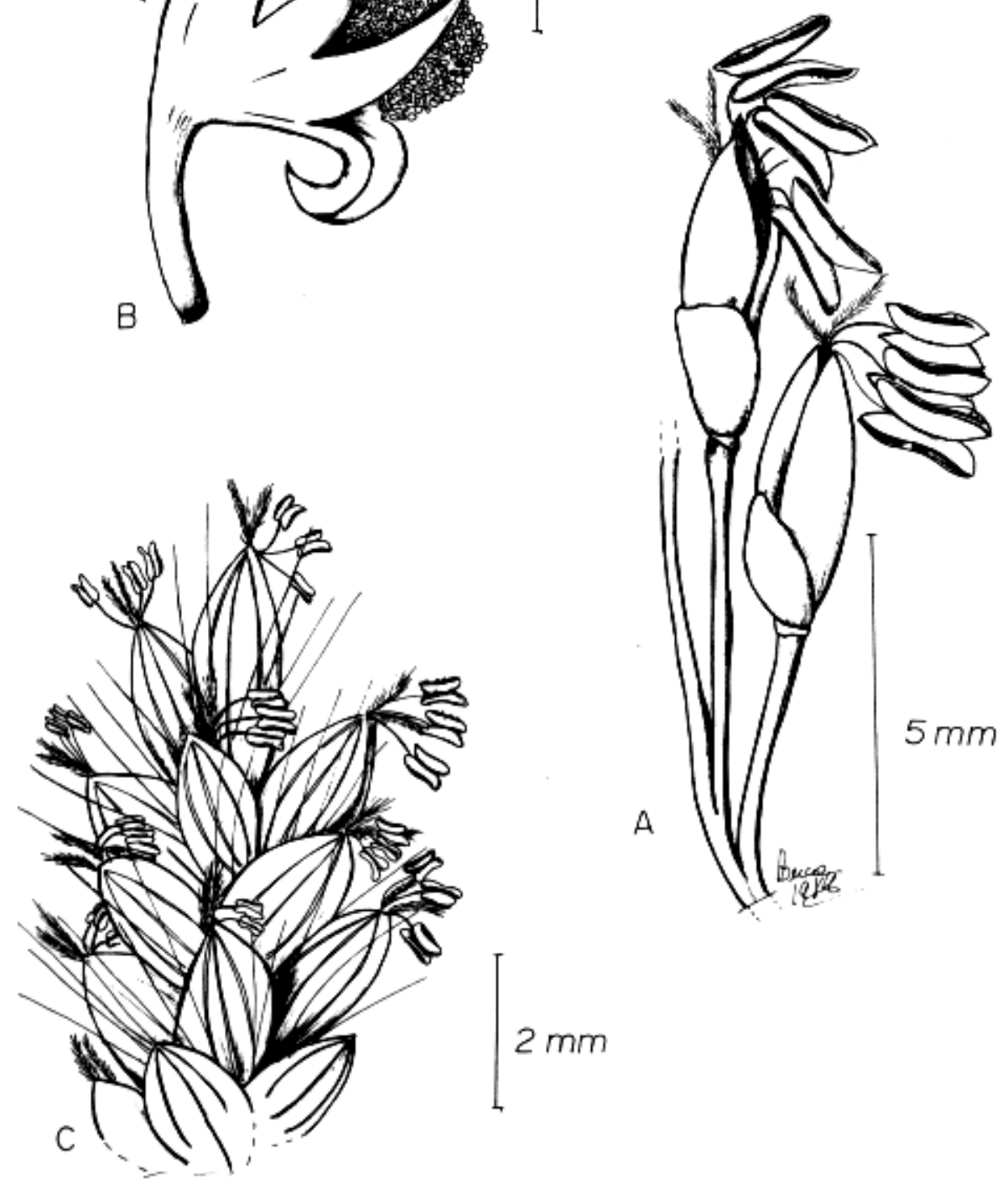

FIGURA 6 - A - Detalhe da inflorescēncia de Panicum maximum, B - inflorescēncia de Ricinus communis, C - detalhe da inflorescēncia de Setária geniculata. 

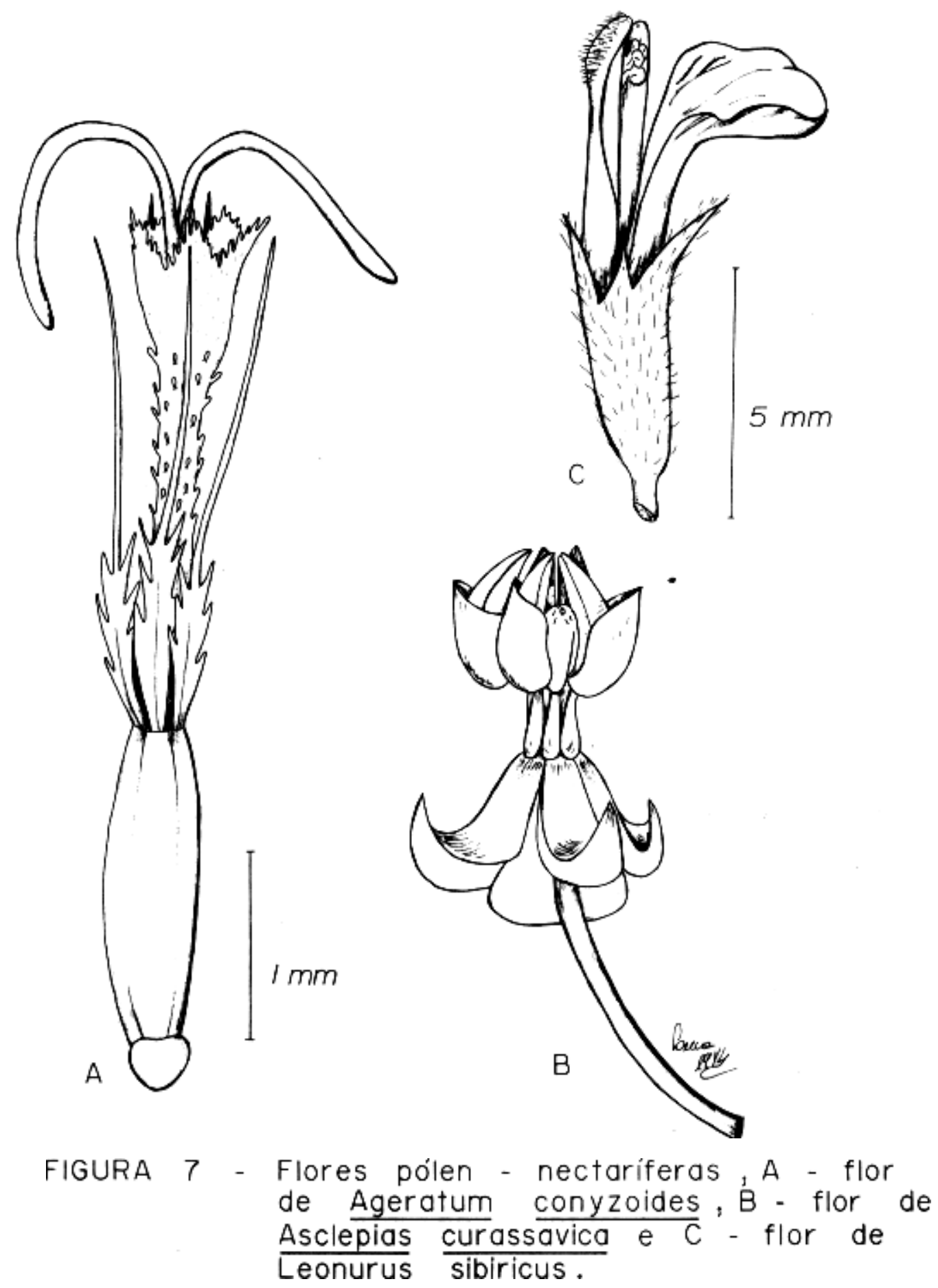

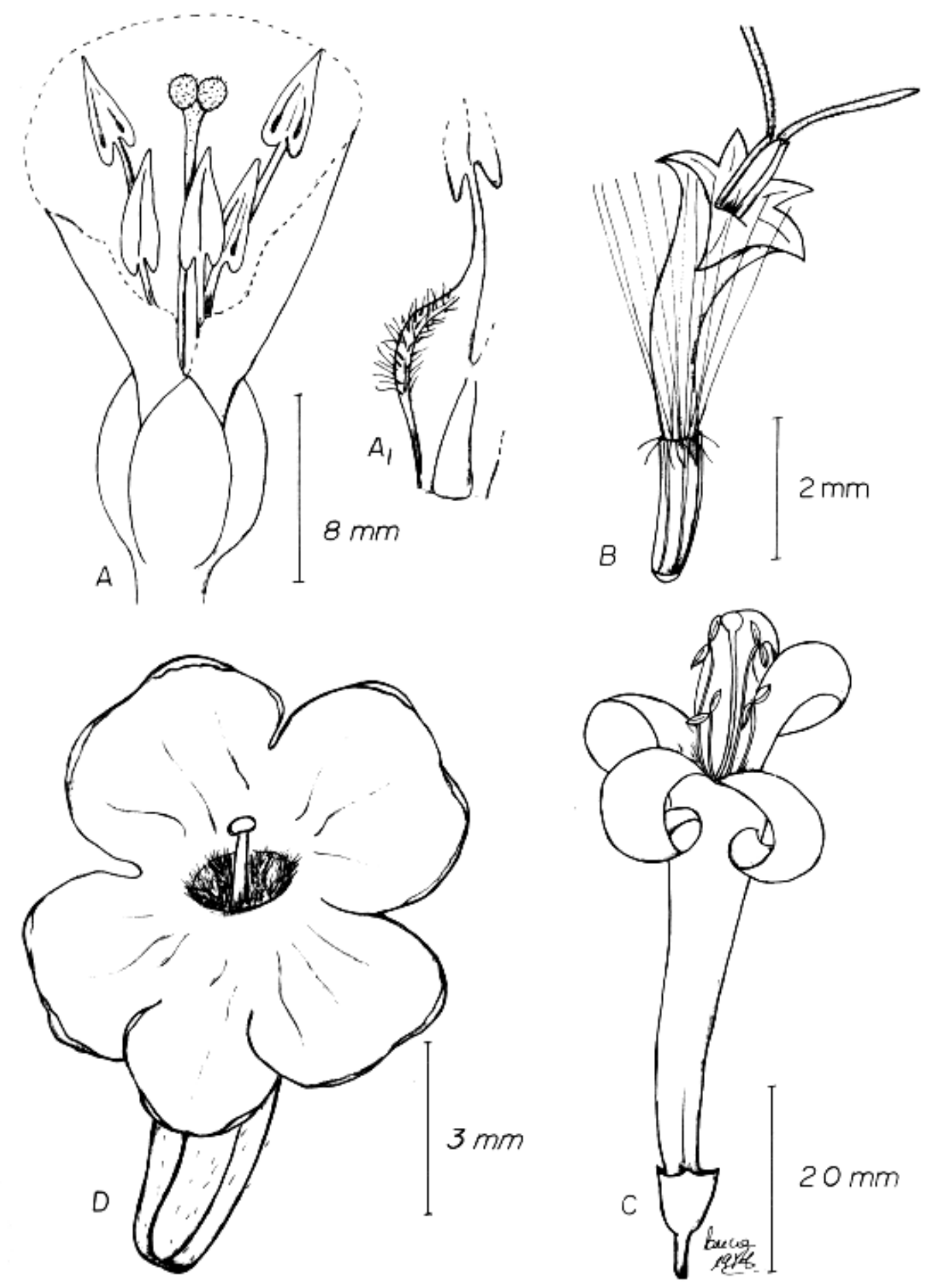

FIGURA 8 - Flores pólen - nectariferas - A - Deta the interno, $A_{l}$ - detalhe da base do estame com pelos nectaríferos de Ipomoea cairica, B - flor de Mikania

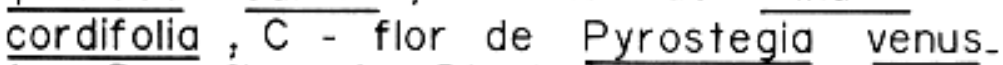
ta:D - flor de Stachy farphetta cayenen sis. 


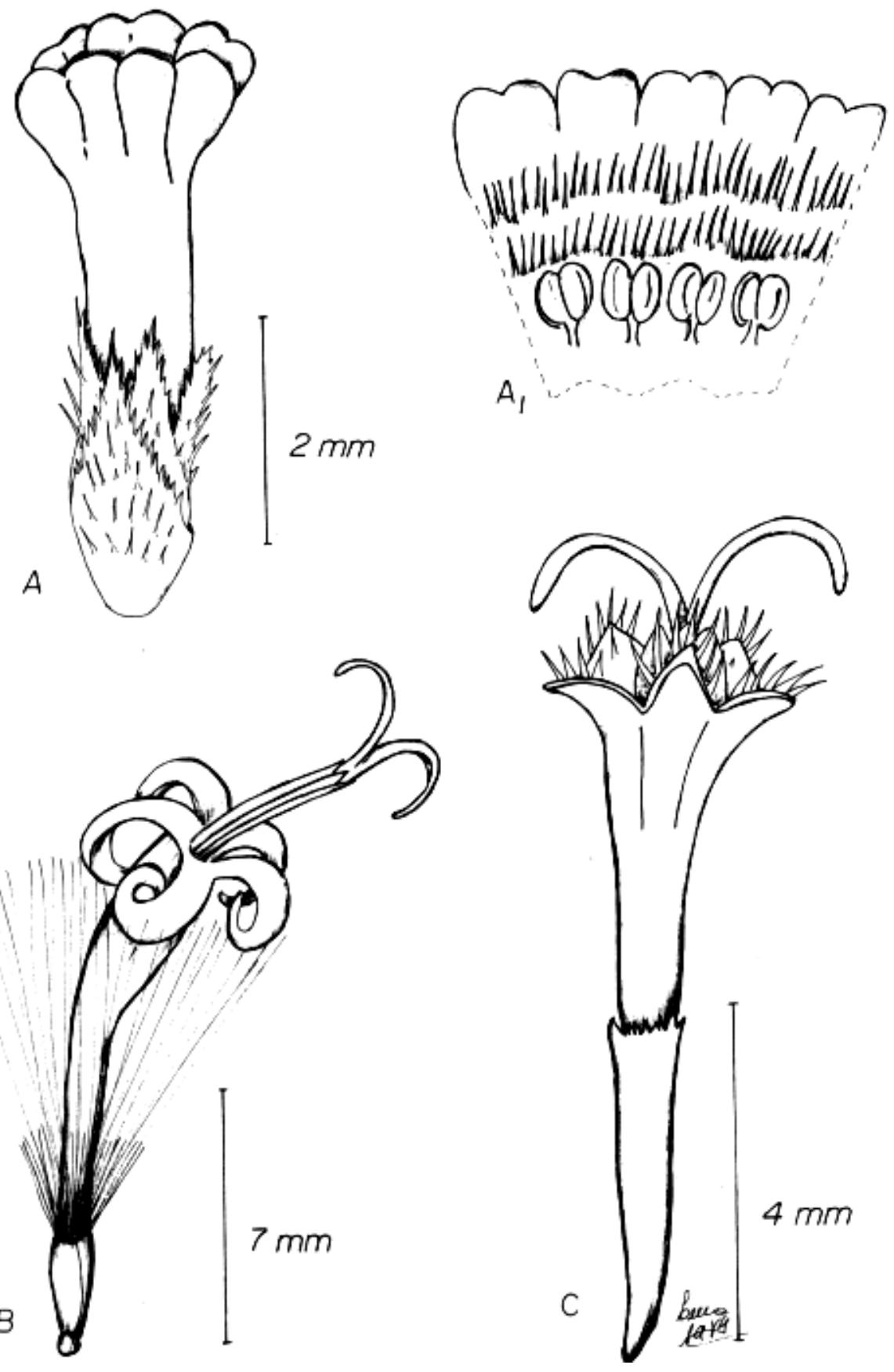

FIGURA 9 - Flores pólen - nectaríferas; A - flor e $A_{1}$ detalhe interno de Verbena litoralis $B$ - flor de Vernonia ferruginea e C - flor de Wedelia paludosa. 
dos em 34 famílias (tabela 2)

As figs. 1 a 9, mostram tipos diver sos de flores, e nectários de algúmas das espécies catal ogadas.

Observa-se qúe as abelhas são bastante volúveis na escolha de plan tas de súa preferência. Múitas vezes deixam plant as sob útilização e ainda em processo de florescimento (plantas de floração longa e initer rúpta) e procuram novas fontes, no momento, em disponibilidade. Muitas vezes, essas novas fontes possuem floração intensa, com períodosde curta duração. Espécies distintas podem então ser vistas, ao mesmo tempo, sobre a mesma espécie vegetal, atropelando-se mutuamente. Essas observações foram feitas a nível de campo com plantas dos gêneros: Hyptis (Labiatae) e Baccharis (Compositae). Esgotadas essas novas fontes regressam às fontes primitivas. As espécies observadas pertencem aos gêneros: Apis, Bombus, Trigona, Tetragonis ca, Partamona e Melipona.

As plantas daninhas, vistas como fornecedoras de néctar e pólen, apresentam muitas vantagens sobre as plantas nativas ou cultivadas. Devido a sua grande adaptabilidade às condições edafoclimáticas marcam presença em todas as regiões fisiográficas do Estado. Geralmente, pertencem à famílias bastante evolui das, que apresentam meios de dispersão sofisticados (1).

Em função desse germoplasma tão adaptável, as plantas daninhas, implantamse e desenvolvem-se com facilidade, sendo portanto, possíveis de serem cultivadas econo micam ente . Mesmo quando ceifadas ou injuriadas reagem satisfatoriamente, antecipando sua floração e produzindo unidades de dispersão viáve is.

Esse comportamento poderia ser explorado, quando de um cultivo racional, visan do o fornecimento de néctar e pólen em perío dos de escas sez.

Por outro lado, o cultivo dessas plantas, combatidas como daninhas nas lavouras, viria a proporcionar condições de manutenção desses agentes considerad os polinizadores objetivando, consequentemente, maior produção das culturas de interes se agropecuário.

\section{CONCLUSÕES}

- Foram listadas 164 espécies, inclusas em 92 gêneros, englobando 34 famílias.

- Encontraram-se 29 espécies que flore scem todo o ano, sendo 9 espécies fornecedoras de pólen, 12 espécies fornecedoras de néctar e 8 espécies fornecedoras de pólen e néctar.

\section{REFERÊNCIAS}

1. Brandão, M.B. \& Sou sa Cúnha, L. H. de, Meios de dispersão em pregados por plantas dani nhas Informe Agropecuário, 8 (87): 4-10 1982.

2. Cardenas, J .; Reyes, C.E. \& Doll, J.D. Tropical Weeds. 
1a ed., Bo gotá, Li brary of Cong ress, 1972. 335 p. il.

03. Campelo, C.R. Es tu do das plantas né ct ar-po li nífe ras (a pícolas) na Baix ad a Fluminense. In: CONGRESSO NACIONAL DE BOTÂNICA, 28 0, Garan hus, 1972. An ais, pp. 293-298.

5. Fe rreira, M.B. \& Laca-Buen dia, J.P. Plantas daninhas em áreas cú ltivad as no Estado de Minas Ge ra is. Plan ta Da ninha, 01 (2): 16-26, 1978 .

6. Fe rreira, M. B. Plantas apícolas no Es ta do de Minas Gerais. In forme Ag ro pecuário. 7 (7 5): 40 -47, 1981 .

4. Kerr, W.E. Comece certo com as abel has. Cooper co ti a, 22 1: 28 36,1968 .
7. Le it ão Filho, H. de F.; Aranha, C. \& Bacchi. 0. Plantas in vaso ras de culturas no Es ta do de São Paúlo. São Paulo. HU CI TEC, 1972. $289 \mathrm{p}$.

8. Le it ão Filho, H. de F.; Aranha; C. $\&$ Bacchi. Plantas invasoras de cultúr as no Es tado de São Paúlo, II. São Paulo. AGI PL AN. 1975. 597p .

10. Lorenzi, H. Plantas daninhas do Brasil: Terrestres, aquáticas, parasitas, toxicas e medicinais. Nova Odessa, Ed. do Autor, 1982. $420 \mathrm{p}$.

12. Vi eira, M.I. Criar abel has e lucro certo. Manual Prático.S ão Paulo, Livraria Nobel, $1983,175 \mathrm{pp}$. 